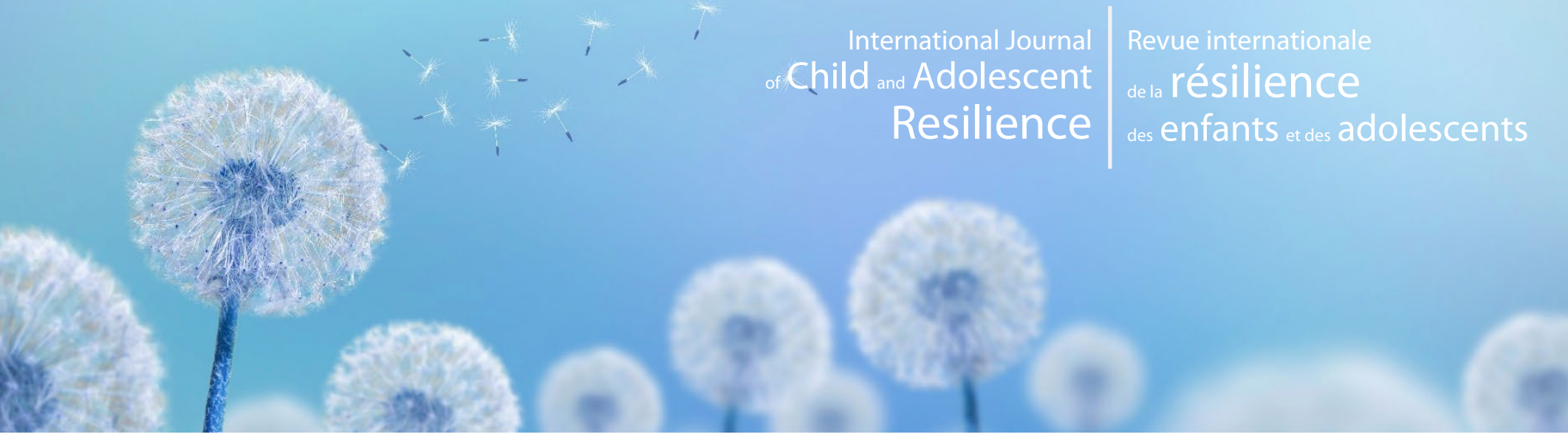

\title{
Resilience in Nicaragua: Preliminary Factor Analysis and Validation of the CYRM-28
}

\section{B. Cameron STUMPF ${ }^{1}$ and Kelly B. T. CHANG ${ }^{1}$}

1 Department of Psychology, George Fox University

Corresponding Author: Kelly B.T. Chang, Ph.D., Department of Psychology, George Fox University, 414 N. Meridian St., PO Box 6127, Newberg, OR 97132.

Email: kchang@georgefox.edu

\section{Abstract}

Objectives: The current study used factor analytic techniques to examine the performance of the Child and Youth Resilience Measure (CYRM-28) in Nicaragua.

Methods: Nicaraguan youth from Managua, León, Chinandega, and Granada ( $n=2,764)$ completed the CYRM-28 and the Personal Wellbeing Index (PWI-A). The CYRM-28 and PWI-A are self-report measures that were translated into Spanish in previous studies.

Results: A confirmatory factor analysis on the CYRM-28 did not support the eight-factor model from previous research. An exploratory factor analysis yielded a six-factor model: Social Belonging, Cultural Context, Caregiver Context, Social Skills, Spiritual Context, and Responsibility. Seven items were removed to improve model fit, reducing the CYRM-28 to only 21 items. We named the reduced measure the CYRM-21-N (Nicaragua).

Implications: From our analyses, we constructed the CYRM-21-N, a briefer version of the CYRM-28 with a revised factor structure. The CYRM-21-N may be useful in studying resilience in youth populations or assessing intervention outcomes in Nicaragua.

Keywords: Resilience; CYRM-28; youth; Nicaragua; subjective well-being. 


\section{Introduction}

While happiness is almost universally sought, researchers have struggled to precisely define it and measure it. Consequently, subjective well-being (SWB) was proposed as a broad construct through which emotions, domain satisfaction, and life satisfaction might be assessed (Diener et al., 1999). SWB has been implicated as a potential component for positive youth development (Bird \& Markle, 2012) and has been associated with health, longevity, and relational success (Diener et al., 2018). However, SWB, similar to happiness, can be an elusive and challenging target. For this reason, research is increasingly focusing on the role resilience plays in the achievement and maintenance of SWB in youth populations, especially in countries like Nicaragua.

\section{Resilience}

Resilience can be broadly defined as the ability to adapt to and overcome challenges that arise in life (Fleming \& Ledogar, 2008). These challenges could include the loss of family members, job termination, or even war. The literature is full of many other definitions of resilience, likely a result of varying cultural definitions. However, most definitions include elements of both internal and external resources (Liebenberg \& Joubert, 2019). Internal resources can include self-efficacy, problem-solving skills, and self-control. External resources are both relational and cultural/contextual and can include caregiver support, economic factors, and education. Some resources, such as faith and spirituality, transcend these boundaries and are both internal and external.

Research suggests that resilience may help to cultivate SWB. For example, psychological resilience may play a mediating role between spontaneous activity in the left orbitofrontal cortex and affect balance (Kong et al., 2018). Increased spontaneous activity in this area has been observed in individuals with schizophrenia, bipolar disorder, and major depressive disorder (Chang et al., 2019). These results suggest that greater resilience may provide benefits in the form of affect regulation and symptom reduction, which could then have a positive cascade effect on the other components of SWB. That is, individuals suffering from depression may be "lifted out" of it to some degree by the presence of resilience factors, which could then improve their prognosis.

Resilience may also help in the maintenance of SWB, especially when considering individuals with posttraumatic stress disorder (PTSD). Zahradnik et al. (2010) noted that Nova Scotian Mi'kmaq youth who were exposed to violence were likely to develop PTSD reexperiencing symptoms. However, this only held for those with lower levels of resilience. Youth who scored higher on the global, community, and family levels of resilience showed no increase in PTSD reexperiencing symptoms as a function of exposure to violence. These results demonstrate one of the fundamental aspects of resilience: the ability to overcome adversity (Rutter, 1990). In this way, resilient people may be more likely to maintain higher levels of SWB as a result of their ability to endure hardship.

Resilience is an increasingly popular component of psychological interventions because it is flexible and can be increased (Masten, 2014). Unfortunately, the flexibility of resilience may also allow for decreases over time. Villora et al. (2020) hypothesized that resilience would moderate the relationship between bullying victimization and SWB in their student population. However, the moderation they observed was quite weak and victims of bullying generally had lower resilience. The researchers noted how the experience of having been bullied may actually have reduced the resilience of these students, given the socially embedded nature of resilience and the damage to social resources that result from bullying. More research is needed on resilience trajectories, and this research certainly does not provide causal evidence, but this serves as a sobering reminder that resilience likely does not provide absolute protection. The individual components of resilience should still be nurtured continuously to reap the full benefits.

\section{Resilience Across Cultures}

Ungar and Liebenberg (2013) have called for culturally-sensitive conceptualizations of resilience, especially when constructing intervention strategies or public policy. As a result, resilience has been examined in a vast array of countries and cultures such as South Africa (Govender et al., 2017; van Rensburg et al., 2019), China and Taiwan (Li \& Yang, 2016; Mu \& Hu, 2016), and indigenous Nova Scotian communities (Zahradnik et al., 2010). Though the presentation of resilience looked different across these contexts, both internal and external resources were generally essential. The specific resources within those broad categories and their relative importance are what varied considerably.

Resilience can be particularly beneficial to populations experiencing high degrees of adversity, such as Nicaraguans. Findings across the research in Nicaragua suggest that they face a significant amount of difficulty. 
Nicaraguans experience high rates of suicidal behavior (Guillén et al., 2015; Rodríguez et al., 2006). Guillén et al. found that the presence of stressful life events (SLEs), such as abuse or death of a loved one, increased suicide rates in adult women. Lack of social support was also a contributing factor. Rodríguez et al. did not find an association between suicidal behavior and sociodemographic factors but did find an association between suicidal behavior and spousal suicidal behavior. Their results suggest that even proximity to others who are experiencing distress may be detrimental to SWB in Nicaragua. According to Cox (2012), marginalized groups (such as those in poverty) in Nicaragua experience lower levels of SWB. This was seen across both urban and rural populations. Cox also found that social support and objective income were predictors of SWB.

From here, two routes towards increasing SWB become apparent: reduce the amount of SLEs directly or help people to cope with these difficulties by promoting resilience. While both routes are valuable in different ways, the present study focuses on the latter. However, culturally diverse research on resilience is still emerging and little research on resilience in Nicaragua has been done, particularly on youth. The present study seeks to expand research on resilience in a youth context in Nicaragua, providing researchers with a foundation that can be used to generate more effective intervention strategies.

\section{Current study}

To this end, we examined the validity of the Child and Youth Resilience Measure (CYRM-28; Ungar \& Liebenberg, 2011). The CYRM-28 was developed by an international team of researchers using quantitative and qualitative methods. Participants were sampled from 11 different countries: The Gambia, Russia, Tanzania, India, Canada, South Africa, Palestine, China, United States, Israel, and Colombia. Soon after, Liebenberg et al. (2012) validated the CYRM-28 for use among Canadian youth. They identified a hierarchical factor structure of three higher-order factors, consisting of eight primary factors. Under the Individual category were Personal Skills, Peer Support, and Social Skills. Under the Caregiver category were Physical Caregiving and Psychological Caregiving. Under the Context category were Spiritual Context, Educational Context, and Cultural Context.

Since it's conception, the CYRM-28 has been used and adapted for use in a variety of countries (see Table 1). Though the CYRM-28 has been validated in several other countries, to our knowledge it has not yet been systematically examined in Nicaragua with a large and varied sample. In this study, we used factor analytic techniques to examine the underlying factors of resilience. We expected the factors to differ from the factors found in Western countries. We also sought to establish predictive validity by investigating whether resilience was associated with SWB in our sample.

\section{Method}

\section{Participants}

Participants were sampled from four large municipalities: Managua, León, Chinandega, and Granada. Participants consisted of 1,108 students from four universities and 1,656 students from seven high schools, aged 1122 from Nicaragua $(M=16.63, S D=2.85)$. The sample included more women $(60 \%)$ than men $(40 \%)$. Most of the participants $(99 \%)$ reported Spanish as their first language. Regarding ethnicity, participants reported being of mixed ethnicity (62\%), Criollo (2\%), Indigenous (1\%), European (1\%), and other (1\%). Many participants (33\%) reported not knowing.

\section{Procedure}

Institutional Review Board approval was granted from George Fox University in the United States and from Universidad Nacional Autónoma de Nicaragua. Permission was obtained from directors of the school programs to apply paper-and-pencil questionnaires in the classrooms, and informed consent was gathered from classroom instructors and participants.

The questionnaires were first analyzed by several experts, including Nicaraguan psychology faculty, a professional translator of Nicaraguan origin, and students in the pilot study. The psychology faculty were consulted in several meetings, examining the instructions, questions, and answer format of each questionnaire, as well as the relevance of each construct in this culture. The selected questionnaires had previously been translated, back-translated, and validated with a Spanish-speaking population, whether in the United States, Spain, or a Latin American country like Colombia (Alfaro et al., 2014; Ungar \& Liebenberg, 2011). The Nicaraguan translator made minor corrections and adjustments, following instructions to make the questionnaire format consistent across scales. 
Table 1. Cross-Cultural Comparison of CYRM Factor Structure

\begin{tabular}{|c|c|c|c|}
\hline Samples/Factor Models & Item Count & $a^{a}$ & Study \\
\hline Nova Scotian Mi'kmaq $(n=126)$ & 26 & & Zahradnik et al., 2010 \\
\hline Individual & 9 & .79 & \\
\hline Family & 6 & .72 & \\
\hline Community & 11 & .86 & \\
\hline Columbia $(n=148)$ & 7 & & Montoya et al., 2011 \\
\hline Canada $(n=410)$ & 28 & & Liebenberg et al., 2012 \\
\hline Individual ${ }^{b}$ & 11 & $.65-.91$ & \\
\hline Caregiver $^{b}$ & 7 & $.65-.91$ & \\
\hline Context ${ }^{b}$ & 10 & $.65-.91$ & \\
\hline Canada $(n=589)$ & 27 & & Daigneault et al., 2013 \\
\hline Individual/Social & 11 & .84 & \\
\hline Family & 7 & .78 & \\
\hline Community/Spiritual & 6 & .64 & \\
\hline China $(n=2,632)$ & 12 & .83 & Mu \& Hu, 2016 \\
\hline Tehran $(n=412)$ & 28 & & Amirsardari et al., 2016 \\
\hline Individual & 11 & .78 & \\
\hline Caregiver & 7 & .80 & \\
\hline Context & 10 & .77 & \\
\hline South Africa ( $n=1,854)$ & 24 & & Govender et al., 2017 \\
\hline Individual/Social & 11 & .82 & \\
\hline Familial & 7 & .71 & \\
\hline Community/Spiritual & 6 & .70 & \\
\hline New Zealand $(n=297)$ & 28 & & Sanders et al., 2017 \\
\hline Individual & 7 & .66 & \\
\hline Family & 7 & .81 & \\
\hline Social/Cultural Context & 10 & .77 & \\
\hline Spiritual/Community Context & 4 & .75 & \\
\hline $\operatorname{Iran}(n=353)$ & 11 & & Zand et al., 2017 \\
\hline Peer & 2 & .82 & \\
\hline Caregiver & 4 & .70 & \\
\hline Religious/Cultural & 5 & .72 & \\
\hline Australia $(n=233)$ & 28 & & Langham et al., 2018 \\
\hline Sources & 15 & - & \\
\hline Expressions & 13 & - & \\
\hline Syria $(n=324)$ & 12 & & Panter-Brick et al., 2018 \\
\hline Individual & 4 & - & \\
\hline Relational & 4 & - & \\
\hline Contextual & 4 & - & \\
\hline Jordan ( $n=279)$ & 12 & & Panter-Brick et al., 2018 \\
\hline Individual & 4 & - & \\
\hline Relational & 4 & - & \\
\hline Contextual & 4 & - & \\
\hline Jamaica $(n=244)$ & 28 & & Craig et al., 2018 \\
\hline Individual & 8 & .73 & \\
\hline Social & 10 & .77 & \\
\hline Community & 4 & .77 & \\
\hline Family/Spiritual & 6 & .81 & \\
\hline Spain $(n=432)$ & 32 & & Llistosella et al., 2019 \\
\hline Individual Skills & 15 & .78 & \\
\hline Interaction w/ Family & 7 & .79 & \\
\hline Interaction w/ Others & 10 & .72 & \\
\hline South Africa ( $n=1,137)$ & 28 & & van Rensburg et al., 2019 \\
\hline Individual & 7 & $.70 / .66$ & \\
\hline Family/Relational & 7 & $.80 / .82$ & \\
\hline Contextual ${ }^{\mathrm{b}}$ & 14 & $.82 / .81$ & \\
\hline
\end{tabular}

Note. ${ }^{a}$ Dashes indicate missing reliability information. ${ }^{b}$ Factors contain subfactors or item parcels. 
The pilot sample, consisting of 28 university and 24 high school students, confirmed that the questionnaires were understandable and clear.

In 2015, 3,322 university and high school students completed the measures in their classrooms. In larger schools, classrooms were randomly selected. In smaller schools, all classrooms were selected. University classrooms required instructor permission. Attempts were made to sample a variety of majors. Data from one university were collected via the internet $(n=189)$. After excluding participants who were above 22 years of age, below 11 years of age, or responded carelessly, 2,764 participants remained for the analyses.

\section{Measures}

Resilience. The Child and Youth Resilience Measure (CYRM-28; Ungar \& Liebenberg, 2011) is widely used for measuring resilience in youth populations and is available in several languages. This 28 -item scale includes items regarding a participant's perceptions of their skills, culture, and social support. An example item is: "I am aware of my own strengths." Participants are asked to rate on a scale from 1 to 5 how well they agreed with each item (not at al/to a (ot). The 28 items can either be summed into an index score or decomposed into specific factor mean scores. The Spanish version was developed in Colombia during the original project (Ungar \& Liebenberg, 2011). Studies using the CYRM-28 in a variety of countries have identified factor structures that deviated from the Canadian sample (Liebenberg et al., 2012). The CYRM-28 factors identified in this study demonstrated a range of reliability estimates ${ }^{1}$ in this sample $(a=.56-.73, \omega=.57-.75)$.

Subjective Well-Being (SWB). The Personal Wellbeing Index (PWI-A; International Wellbeing Group, 2006; Lau et al., 2005) is a common measure of general SWB. The Spanish translation has been validated with adolescents in Chile (Alfaro et al., 2014). The 7-item scale asks participants to rate their satisfaction in different life domains: health, personal safety, personal relationships, future security, standard of living, life achievement, and communityconnectedness. An example item asks: "How satisfied are you with your health?" Responses range from 0 (No satisfaction at al/ to 10 (Complete satisfaction). Following the recommendations of Sarriera et al. (2014), two other items were considered for inclusion with the original seven domains of satisfaction on the PWI-A. Satisfaction with spirituality contributed to psychometric performance, while satisfaction with religion did not. Therefore, spirituality was summed with the original seven items into a single index score composed of eight items. The PWI-A index demonstrated strong reliability in this sample $(a=.84, \omega=.84)$.

\section{Analysis}

All analyses were completed in JASP (JASP Team, 2020). Hierarchical confirmatory factor analyses were conducted as SEM models using maximum likelihood estimation. In addition to chi-square $\left(x^{2}\right)$, the following fit indices were used: the comparative fit index (CFl; general cut-off $\geq .90$ ); the Tucker-Lewis Index (TLI; general cut-off $\geq .90$ ); Bayesian information criterion (BIC; smaller values indicate better fit); expected cross-validation index (ECVI; smaller values indicate better fit); the root mean square error of approximation (RMSEA; general cut-off $\leq .06$ ). The BIC and ECVI indices are useful for comparing non-nested models. Interpretations of fit indices follow recommendations by West et al. (2012), including a warning against treating cut-off values as objective standards.

\section{Results}

Missing values for the CYRM-28 and PWI-A were minimal (1-3\%; see online supplemental materials at https://osf.io/9hg48/). Listwise deletion was applied to participants with more than 20\% missing data (Peng et al., 2006). The remaining missing values were imputed via the linear trend at point method. Descriptive statistics are shown in Table 2. CYRM-28 index scores were normally distributed (skewness $=-0.33$, kurtosis $=0.37$ ), while PWI-A index scores displayed more significant deviation from normality (skewness $=-1.27$, kurtosis $=2.06$ ). The data were appropriate for factor analysis, as indicated by the Kaiser-Meyer-Olkin (KMO) measure, $\mathrm{KMO}=.91$, and Bartlett's Test of Sphericity, $X^{2}(378)=119075.33, p<.001$. Model loadings, covariance/residual matrices, and modification indices are available in the online supplemental materials.

\footnotetext{
1 The consequences of this are considered in the discussion.
} 
Table 2. Descriptive Statistics for PWI-A and CYRM-21-N Factor Scores

\begin{tabular}{lcccc}
\hline \multicolumn{1}{c}{ Scale } & $M$ & $S D$ & Range & Reliability $(\alpha / \omega)$ \\
\hline PWI-A & 64.87 & 12.42 & $0-80$ & $.84 / .84$ \\
CYRM-21-N & & & & $.85 / .85$ \\
$\quad$ Belonging & 3.51 & 0.77 & $1-5$ & $.71 / .72$ \\
Family & 3.67 & 0.85 & $1-5$ & $.73 / .75$ \\
Social Skills & 3.89 & 0.63 & $1.5-5$ & $.59 / .60$ \\
Spiritual Context & 3.57 & 0.86 & $1-5$ & $.64 / .66$ \\
Cultural Context & 4.01 & 0.72 & $1-5$ & $.57 / .59$ \\
Responsibility & 3.82 & 0.72 & $1-5$ & $.56 / .57$ \\
\hline
\end{tabular}

Note. CYRM factors shown are from the 21-item scale shown in Table 4

\section{Factor Analyses of the CYRM-28}

Three hierarchical confirmatory factor analyses were conducted (CFA; see Table 3). The first CFA (Model A) did not confirm the eight-factor model, $\mathrm{X}^{2}(339)=3491.01, p<.001, \mathrm{CFI}=.82, \mathrm{TLI}=.80, \mathrm{BIC}=209338.62$, ECVI $=1.33$, RMSEA $(90 \% \mathrm{Cl})=.058(.057, .060)$. Next, an exploratory factor analysis using principal component analysis extraction and varimax rotation was conducted. The resulting six factors with eigenvalues above 1.00 were identical to those identified by Chang and Buckles (2018), and as such we retained the factor names they employed: Belonging, Cultural Context, Caregiver Context, Social Skills, Spiritual Context, and Responsibility.

The six-factor model (Model B) fit the data better than the eight-factor model, but still failed to fit adequately, $X^{2}(341)=3240.77, p<.001, \mathrm{CFI}=.84, \mathrm{TLI}=.82, \mathrm{BIC}=209072.57, \mathrm{ECVI}=1.24, \mathrm{RMSEA}(90 \% \mathrm{Cl})=.056(.054, .058)$. Model B was examined in more detail using modification indices, factor loadings, and residuals. The following item pairs showed large covariation unaccounted for in the model: 26 and 27;5 and 6;9 and 23. Item loadings for items 1, 4, 7, and 13 fell below. 40. An inspection of the residuals suggested that items 3 and 15 were contributing to poor model fit. For these reasons, as well as theoretical reasons expanded upon in the Discussion, items 1, 3, 5, 7, 13, 15, and 26 were removed from the analyses. The remaining items constitute what we call the CYRM-21-N (Nicaragua).

Model B was examined a second time using only the remaining 21 items (Model C; see Table 4). Additional covariances were added, as suggested by Mls for Model B. This involved freeing the covariances between items 9 and 22,9 and 23, and 14 and 18. The semantic similarity of these items supported this decision on theoretical grounds. Fit for Model $\mathrm{C}$ was adequate, $\mathrm{X}^{2}(177)=1182.16, p<.001, \mathrm{CFI}=.92, \mathrm{TLI}=.91, \mathrm{BIC}=155199.65, \mathrm{ECVI}=0.47, \mathrm{RMSEA}(90 \% \mathrm{Cl})$ $=.046(.043, .048)$. The BIC and ECVI indices support the superiority of Model C over the two alternatives. We continued to retain the factor names identified by Chang and Buckles (2018), as the factors were thinned but not substantially changed. These factors produced a range of reliability estimates $(\alpha=.56-.73, \omega=.57-.75)$. Higher reliability estimates were found when combining the 21 remaining items into a composite index score $(a=.85, \omega=.85)$.

Table 3. Results of the Confirmatory Factor Analyses of the CYMR-28 $(n=2,727)$

\begin{tabular}{|c|c|c|c|c|c|c|c|c|c|}
\hline \multirow[t]{2}{*}{ Model $^{a}$} & \multirow[t]{2}{*}{$x^{2}$} & \multirow[t]{2}{*}{$d f$} & \multirow[t]{2}{*}{ CFI } & \multirow[t]{2}{*}{ TLI } & \multirow[t]{2}{*}{$\mathrm{BIC}$} & \multirow[t]{2}{*}{ ECVI } & \multirow[t]{2}{*}{ RMSEA } & \multicolumn{2}{|c|}{$95 \% \mathrm{Cl}$} \\
\hline & & & & & & & & $\angle L$ & $U L$ \\
\hline A: 8-Factor ${ }^{b}$ & $3491.01^{* * *}$ & 339 & .82 & .80 & 209338.62 & 1.33 & .058 & .057 & .060 \\
\hline B: 6-Factor ${ }^{b}$ & $3240.77^{* * *}$ & 341 & .84 & .82 & 209072.57 & 1.24 & .056 & .054 & .058 \\
\hline C: 6-Factor ${ }^{c}$ & $1182.16^{* * *}$ & 177 & .92 & .91 & 155199.65 & 0.47 & .046 & .043 & .048 \\
\hline
\end{tabular}

Note. Structural equation modeling was used for the analysis. CFI = comparative fit index; TLI = Tucker-Lewis Index; $\mathrm{BIC}=$ Bayesian information criterion; $\mathrm{ECVI}=$ expected cross-validation index; RMSEA = root mean square error of approximation; $\mathrm{Cl}=$ confidence interval; $L L=$ lower limit; $U L=$ upper limit.

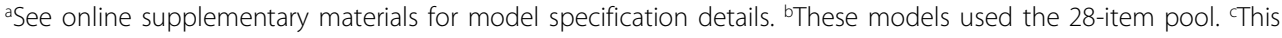
model used the 21 -item pool.

${ }^{* * *} p<.001$. 
Table 4. Factor Loadings for the CYRM-21-N $(n=2,727)$

\begin{tabular}{|c|c|c|c|c|c|c|}
\hline \multirow[t]{2}{*}{ Factors/Items } & \multicolumn{6}{|c|}{ Factor Loading } \\
\hline & 1 & 2 & 3 & 4 & 5 & 6 \\
\hline \multicolumn{7}{|l|}{ Belonging } \\
\hline 11. People think that I am fun to be with. & .49 & & & & & \\
\hline 14. I feel supported by my friends. & .55 & & & & & \\
\hline 16. I feel I belong at my school. & .67 & & & & & \\
\hline 18. My friends stand by me during difficult times. & .52 & & & & & \\
\hline \multicolumn{7}{|l|}{ Cultural Context } \\
\hline 10. I am proud of my ethnic background. & & .64 & & & & \\
\hline 27. I enjoy my community's traditions. & & .58 & & & & \\
\hline 28. I am proud to be a citizen of Nicaragua. & & .45 & & & & \\
\hline \multicolumn{7}{|l|}{ Family } \\
\hline 6. My parent(s)/caregivers(s) know a lot about me. & & & .62 & & & \\
\hline 12. I talk to my family/caregiver(s) about how I feel. & & & .57 & & & \\
\hline 17. My family stands by me during difficult times. & & & .56 & & & \\
\hline 24. I feel safe when I am with my family/caregiver(s). & & & .71 & & & \\
\hline \multicolumn{7}{|l|}{ Social Skills } \\
\hline 2. I cooperate with people around me. & & & & .43 & & \\
\hline 4. I know how to behave in different social situations. & & & & .32 & & \\
\hline 8. I try to finish what I start. & & & & .56 & & \\
\hline 21. I am aware of my own strengths. & & & & .63 & & \\
\hline \multicolumn{7}{|l|}{ Spiritual } \\
\hline 9. Spiritual beliefs are a source of strength for me. & & & & & .60 & \\
\hline 22. I participate in organized religious activities. & & & & & .47 & \\
\hline 23. I think it is important to serve my community. & & & & & .77 & \\
\hline \multicolumn{7}{|l|}{ Responsibility } \\
\hline 19. I am treated fairly in my community. & & & & & & .52 \\
\hline $\begin{array}{l}\text { 20. I have opportunities to show others that I am becoming an adult } \\
\text { and can act responsibly. }\end{array}$ & & & & & & .58 \\
\hline $\begin{array}{l}\text { 25. I have opportunities to develop skills that will be useful later in life } \\
\text { (like job skills and skills to care for others). }\end{array}$ & & & & & & .54 \\
\hline
\end{tabular}

Notes. Loadings correspond to those from Model C (see Table 3).

\section{Predicting PWI-A from CYRM-21-N Factors}

A stepwise linear regression was conducted to predict PWI-A scores from the six resilience factors confirmed in Model C (see Table 5). Resilience predicted 25\% of the variance, $F(6,2673)=148.03, p<.001$. All factors were significant predictors of PWI-A scores except for Responsibility. An examination of the residual plot revealed that the assumption of homoscedasticity was violated. This is likely explained by the PWI-A's ceiling effect.

Table 5. Linear Regression Results for PWI-A Scores Predicted by CYRM-21-N Factors

\begin{tabular}{lccrrr}
\hline \multicolumn{1}{c}{ Variable } & $B$ & SE & \multicolumn{2}{c}{$95 \% \mathrm{Cl}$ for $B$} & \multirow{2}{*}{$\beta$} \\
\cline { 4 - 5 } & & & $\mathrm{LL}$ & $\mathrm{UL}$ & \\
\hline Constant & $22.72^{* * * *}$ & 1.56 & 19.66 & 25.79 & \\
Belonging & $3.35^{* * *}$ & 0.33 & 2.70 & 4.00 & $.21^{* * * *}$ \\
Family & $2.42^{* * * *}$ & 0.30 & 1.84 & 3.00 & $.16^{* * * *}$ \\
Social Skills & $3.11^{* * * *}$ & 0.41 & 2.31 & 3.92 & $.16^{* * * *}$ \\
Spiritual Context & $1.27^{* * *}$ & 0.28 & 0.72 & 1.81 & $.09^{* * * *}$ \\
Cultural Context & $0.68^{*}$ & 0.35 & 0.01 & 1.36 & $.04^{*}$ \\
Responsibility & 0.56 & 0.37 & -0.16 & 1.28 & .03 \\
\hline
\end{tabular}

Note. $\mathrm{Cl}=$ confidence interval; $\mathrm{LL}=$ lower limit; $\mathrm{UL}=$ upper limit. ${ }^{*} \mathrm{p}<.05,{ }^{* *} \mathrm{p}<.01,{ }^{* * *} \mathrm{p}<.001$. 


\section{Discussion}

The present study aimed to examine the performance of the CYRM-28 in Nicaraguan youth. A confirmatory factor analysis did not support the eight-factor model from previous research (Liebenberg et al., 2012). An exploratory factor analysis yielded a six-factor model: Social Belonging, Cultural Context, Caregiver Context, Social Skills, Spiritual Context, and Responsibility. However, this model did not adequately explain the covariance structure of the data. After removing several items and reducing the CYRM-28 to a 21-item scale (CYRM-21-N), the model fit was adequate. Furthermore, five of the six factors were significant predictors of SWB.

The removal of seven items was supported empirically by our data analysis. In addition, these choices may be theoretically justified. For instance, Item 13 reads: "I am able to solve problems without harming myself or others (for example by using drugs and/or being violent)." Participants may be misled by the ambiguous wording to think that drugs or violence are examples of solving problems without harming oneself or others, or to think that the question was about whether they use drugs or violence. Additionally, Item 26 reads: "I enjoy my family's/caregiver's cultural and family traditions." Double-barrelled questions like this (e.g., those that ask multiple questions at once) may also be considered confusing to participants.

\section{Features of the CYRM-21-N}

The CYRM-21-N factors demonstrated a broad range of reliability estimates that could potentially be considered problematic. That said, reliability estimates have been poorly understood since their conception (Cho \& Kim, 2015). Peterson (1994) noted how reliability estimates can be biased by the number of items, particularly when there are fewer than four items on a given scale. Given that each of the six factors in the CYRM-21-N has four or fewer items, the low estimates are not surprising. Furthermore, higher reliability is not necessarily desirable for our purposes. Increased reliability results in a decrease in content validity (Cho and Kim, 2015). Additionally, Boyle (1991) argued that low to moderate reliability is actually beneficial to research involving psychological measurements and diverse cultural settings.

In the present sample, five of the six CYRM-21-N factors predicted SWB. These results, although not causal, are consistent with the argument that resilience may help cultivate and maintain SWB. Recall that SWB is invaluable for promoting positive youth development (Bird \& Markle, 2012; Diener et al., 2018). Therefore, the CYRM-21-N may be used to help identify at-risk youth. More specifically, it can help to identify the domains in which they may be lacking the resources to be resilient. This information allows for more personalized intervention strategies, as opposed to a one-size-fits-all approach. Finally, the instrument may also be used to assess the outcomes of resilience-based interventions programs.

The CYRM-21-N has a few advantages over the CYRM-28 for use in Nicaragua. First, its revised factor structure can more accurately be used to differentiate between varying aspects of resilience. Second, the $25 \%$ reduction in length will lessen the burden of responding for participants, especially if other measures are administered in conjunction with the CYRM-21-N. Lastly, the removal of difficult-to-understand and poorly performing items increases the validity of the measure for use in Nicaragua. The CYRM-28 have been examined in South Africa (Govender et al., 2017; van Rensburg et al., 2017), Colombia (Montoya et al., 2011), China (Mu \& Hu, 2016), New Zealand (Sanders et al., 2017), and Iran (Zand et al., 2016), with many of these countries similarly diverging from the eight-factor model and 28-item structure (see Table 1). This underscores the need to evaluate the psychometric properties of instruments before use in cultures in which they were not initially developed.

\section{Resilience Across Culture}

These results, along with the literature, also support the need for culturally-sensitive definitions of resilience as a construct. Factors contributing to resilience differ depending on context and cultural values. In the present study, the factor structure differences that emerged reflected the more collectivist attitude that we would expect to observe in Nicaragua. That is to say, the response pattern in our sample suggests that the participants did not clearly distinguish between personal and community-related domains. Additionally, our results do not support education as a distinct factor. Rather, education is likely tied to other domains of resilience in Nicaragua. For example, Item 16: "I feel I belong at my school," loaded onto the belonging factor.

Understanding resilience in the context of culture is an important step in the intervention process. Nicaragua, for example, faces many challenges associated with lower resilience and well-being (Guillén et al., 2015; Sausner \& 
Webster, 2016). Some of these challenges include political turmoil, poverty, and natural disaster. Understanding and encouraging the development of resilience in the context of their culture may be an effective method of helping individuals to cope. Increasing resilience could have a beneficial effect on the life satisfaction of the country as well (Temiz \& Comert, 2018).

\section{Strengths and Limitations}

The present study supports the ongoing movement towards culturally-appropriate definitions of resilience. Our large sample allowed for sophisticated factor analyses, which in turn provided valuable information about the performance of the CYRM-28 in Nicaragua. This revealed a reduced factor structure. Furthermore, an analysis of these reduced factors indicated that they were useful in predicting well-being in our sample, despite the ceiling effect that occurred in our well-being measure. It is possible that a more robust measure of life satisfaction could yield stronger predictive validity. Regardless, future researchers will now benefit from the availability of a culturally-validated instrument for measuring resilience in Nicaragua.

While the sample for this study is large, it is not a comprehensive representation of Nicaraguan youth. For instance, not all Nicaraguan youth attend educational institutions in large municipalities. Additionally, sampling was not random. Future studies should improve in these areas by sampling larger and more diverse populations using stricter methods. Future studies may also benefit from longitudinal designs examining resilience as a process that follows the presence of certain traits, as suggested by Rutter (1990). Identifying and utilizing these processes may make interventions more effective. Finally, our use of a single sample and model re-specification increased the risk of overfitting. Future studies should seek to empirically confirm the factor structure we identified prior to making use of it.

\section{Implications}

Moving forward, countries like Nicaragua would benefit from research-based intervention strategies. Additionally, the revised CYRM-21-N may be used to assess intervention outcomes in Nicaragua. Ungar and Liebenberg (2013) recommended that these efforts take context and culture into consideration. Much of the language and attitudes surrounding resilience can unintentionally imply that resilience is an individual responsibility, whereas Ungar and Liebenberg argue that it is a societal and cultural responsibility as well. With this in mind, intervention efforts may be augmented with policy changes as well to help lead the Nicaraguan youth towards a state of greater well-being.

\section{Funding}

This project was supported in part by a George Fox University Faculty Grant \#GFU2015G11. Special appreciation to Maria Rosalina Melendez Berrios, Claudia Medina, and Arlen Soto, of Universidad Nacional Autónoma de Nicaragua, León, and Arecely Cardoza of Universidad Cristiana Autónoma de Nicaragua, León for their advice and support.

\section{Conflict of interest}

The authors have no conflict of interest to disclose.

\section{References}

Alfaro, J., Castellá, J., Bedin, L., Abs, D., Casas, F., Valdenegro, B., \& Oyarzún, D. (2014). Adaptación del Índice de Bienestar Personal para adolescentes en lengua española y portuguesa [Adaptation of the Personal Well-Being Index for adolescents on Spanish and Portuguese language]. Universitas Psychologica, 13(1), 239-252. https://doi.org/10.11144/Javeriana.UPSY13-1.aibp

Amirsardari, M., Fathizadeh, S., Tavousi, M., Garmaroudi, G., \& Montazeri, A. (2016). Psychometric properties of International Children and Youth Resilience Measurement (CYRM-28) Scale. Health Education and Health Promotion (HEHP), 4(1), 15-26. http://journals.modares.ac.ir/article-5-5447-en.html

Bird, J. M., \& Markle, R. S. (2012). Subjective well-being in school environments: Promoting positive youth development through evidence-based assessment and intervention. American Journal of Orthopsychiatry, 82(1), 61-66. https://doi.org/10.1111/j.1939-0025.2011.01127.x

Boyle, G. J. (1991). Does item homogeneity indicate internal consistency or item redundancy in psychometric scales? Personality and Individual Differences, 12(3), 291-294. https://doi.org/10.1016/0191-8869(91)90115-R

Chang, K. B. T., \& Buckles, A. A. (2018, April). Validation and factor structure of the Child and Youth Resilience Measure-28 (CYRM-28) 
Resilience in Nicaragua: Preliminary Factor Analysis and Validation of the CYRM-28

among Nicaraguan youth. Poster presented at the Western Psychology Association Convention, Portland, OR.

Chang, M., Edmiston, E. K., Womer, F. Y., Zhou, Q., Wei, S., Jiang, X., Zhou, Y., Ye, Y., Huang, H., Zuo, X., Xu, K., Tang, Y., \& Wang, F. (2019). Spontaneous low-frequency fluctuations in the neural system for emotional perception in major psychiatric disorders: amplitude similarities and differences across frequency bands. Journal of Psychiatry \& Neuroscience, 44(2), $132-141$. https://doi.org/10.1503\%2Fjpn.170226

Cho, E., \& Kim, S. (2015). Cronbach's coefficient alpha: Well known but poorly understood. Organizational Research Methods, 18(2), 207-230. https://doi.org/10.1177/1094428114555994

Cox, K. (2012). Happiness and unhappiness in the developing world: Life satisfaction among sex workers, dump-dwellers, urban poor, and rural peasants in Nicaragua. Journal of Happiness Studies, 13(1), 103-128. https://doi.org/10.1007/s10902-011-9253-y

Craig, L. S., Paisley-Clare, V., Palmer, T. A., \& Govia, I. O. (2018). Validation of the Child and Youth Resilience Measure among Jamaican young adults: Towards the assessment of risk and resilience in a Caribbean population. Caribbean Journal of Psychology, 10(2), 14-40. https://www.uwipress.com/cjp-vol-10-i2-a1/

Daigneault, I., Dion, J., Hébert, M., McDuff, P., \& Collin-Vézina, D. (2013). Psychometric properties of the Child and Youth Resilience Measure (CYRM-28) among samples of French Canadian youth. Child Abuse and Neglect, 37(2-3), 160-171. https://doi.org/10.1016/j.chiabu.2012.06.004

Diener, E., Oishi, S., \& Tay, L. (2018). Advances in subjective well-being research. Nature Human Behaviour, 2(4), $253-260$. https://doi.org/10.1038/s41562-018-0307-6

Diener, E., Suh, E. M., Lucas, R. E., \& Smith, H. L. (1999). Subjective well-being: Three decades of progress. Psychological Bulletin, 125(2), 276-302. https://doi.org/10.1037/0033-2909.125.2.276

Fleming, J., \& Ledogar, R. J. (2008). Resilience, an evolving concept: A review of literature relevant to aboriginal research. Pimatisiwin, 6(2), 7-23. https://journalindigenouswellbeing.com/media/2018/10/3_Fleming.pdf

Govender, K., Cowden, R. G., Asante, K. O., George, G., \& Reardon, C. (2017). Validation of the Child and Youth Resilience Measure among South African adolescents. PLOS ONE, 12(10), e0185815. https://doi.org/10.1371/journal.pone.0185815

Guillén, A. I., Panadero, S., Rivas, E., \& Vázquez, J. J. (2015). Suicide attempts and stressful life events among female victims of intimate partner violence living in poverty in Nicaragua. Scandinavian Journal of Psychology, 56(3), $349-356$. https://doi.org/10.1111/sjop.12207

International Wellbeing Group (2006). Personal Wellbeing Index. Australian Centre on Quality of Life, Deakin University.

JASP Team (2020). JASP (Version 0.12.2) [Computer software]. https://jasp-stats.org/

Kong, F., Ma, X., You, X., \& Xiang, Y. (2018). The resilient brain: Psychological resilience mediates the effect of amplitude of lowfrequency fluctuations in orbitofrontal cortex on subjective well-being in young healthy adults. Social Cognitive \& Affective Neuroscience, 13(7), 755-763. https://doi.org/10.1093/scan/nsy045

Langham, E., McCalman, J., Redman-MacLaren, M., Hunter, E., Wenitong, M., Britton, A., Rutherford, K., Saunders, V., Ungar, M., \& Bainbridge, R. (2018). Validation and factor analysis of the Child and Youth Resilience Measure for Indigenous Australian boarding school students. Frontiers in Public Health, 6, Article 299. https://doi.org/10.3389/fpubh.2018.00299

Lau, A. L. D., Cummins, R. A., \& McPherson, W. (2005). An investigation into the cross-cultural equivalence of the Personal Wellbeing Index. Social Indicators Research, 72(3), 403-430. https://doi.org/10.1007/s11205-004-0561-z

Li, M-H., \& Yang, Y. (2016). A cross-cultural study on a resilience-stress path model for college students. Journal of Counseling \& Development, 94(3), 319-332. https://doi.org/10.1002/jcad.12088

Liebenberg, L., \& Joubert, N. (2019). A comprehensive review of core resilience elements and indicators: Findings of relevance to children and youth. International Journal of Child and Adolescent Resilience, 6(1), 8-18. https://doi.org/10.7202/1069072ar

Liebenberg, L., Ungar, M., \& de Vijver, F. V. (2012). Validation of the Child and Youth Resilience Measure-28 (CYRM-28) among Canadian youth. Research on Social Work Practice, 22(2), 219-226. https://doi.org/10.1177/1049731511428619

Llistosella, M., Gutiérrez-Rosado, T., Rodríguez-Rey, R., Liebenberg, L., Bejarano, Á., Gómez-Benito, J., \& Limonero, J. T. (2019). Adaptation and psychometric properties of the Spanish version of Child and Youth Resilience Measure (CYRM-32). Frontiers in Psychology, 10, Article 1410. https://doi.org/10.3389/fpsyg.2019.01410

Masten, A. S. (2014). Global perspectives on resilience in children and youth. Child Development, 85(1), 6-20. https://doi.org/10.1111/cdev.12205

Montoya, N., Restrepo, A., Duque, L. F., \& Ungar, M. (2011). Predicting resilient adolescents and youths: Validation of a screening test in Colombia. Issues in Comprehensive Pediatric Nursing, 34(4), 167-188. https://doi.org/10.3109/01460862.2011.619400

Mu, G. M., \& Hu, Y. (2016). Validation of the Chinese version of the 12-item Child and Youth Resilience Measure. Children and Youth Services Review, 70, 332-339. https://doi.org/10.1016/j.childyouth.2016.09.037

Panter-Brick, C., Hadfield, K., Dajani, R., Eggerman, M., Ager, A., \& Ungar, M. (2018). Resilience in context: A brief and culturally grounded 
measure for Syrian refugee and Jordanian host-community adolescents. Child Development, 89(5), $1803-1820$. https://doi.org/10.1111/cdev.12868

Peng, C-Y. J., Harwell, M., Liou, S-M., \& Ehman, L. H. (2006). Advances in missing data methods and implications for educational research. In S. Sawilowsky (Ed.), Real data analysis (pp. 31-78). Information Age Publishing Inc.

Peterson, R. A. (1994). A meta-analysis of Cronbach's coefficient alpha. Oxford Journals, 21(2), 381-391. https://doi.org/10.1086/209405

Rodríguez, A., Caldera, T., Kullgren, G., \& Renberg, E. (2006). Suicidal expressions among young people in Nicaragua. Social Psychiatry \& Psychiatric Epidemiology, 41(9), 692-697. https://doi.org/10.1007/s00127-006-0083-x

Rutter, M. (1990). Psychosocial resilience and protective mechanisms. In J. E. Rolf, A. S. Masten, D. Cicchetti, K. H. Nuechterlein, \& S. Weintraub (Eds.), Risk and protective factors in the development of psychopathology (pp. 181-214). Cambridge University Press. https://doi.org/10.1017/CBO9780511752872.013

Sanders, J., Munford, R., Thimasarn-Anwar, T., \& Liebenberg, L. (2017). Validation of the Child and Youth Resilience Measure (CYRM28) on a sample of at-risk New Zealand youth. Research on Social Work Practice, 27(7), 827-840. https://doi.org/10.1177/1049731515614102

Sarriera, J. C., Casas, F., Alfaro, J., Bedin, L., Wachholz Strelhow, M. R., Abs, D., Valdenegro, B., García, C., \& Gyarzún, D. (2014). Psychometric properties of the Personal Wellbeing Index in Brazilian and Chilean adolescents including spirituality and religion. Psicologia: Reflexão e Crítica, 27(4), 710-719. https://doi.org/10.1590/1678-7153.201427411

Sausner, E. B., \& Webster, N. (2016). Migration and community resilience in Nicaraguan Afro-Caribbean coastal communities. Journal of Developing Societies, 32(4), 484-507. https://doi.org/10.1177/0169796X16667876

Temiz, Z. T., \& Comert, I. T. (2018). The relationship between life satisfaction, attachment styles, and psychological resilience in university students. Dusunen Adam Journal of Psychiatry \& Neurological Sciences, 31(3), $274-283$. https://doi.org/10.5350/DAJPN2018310305

Ungar, M., \& Liebenberg, L. (2011). Assessing resilience across cultures using mixed methods: Construction of the Child and Youth Resilience Measure. Journal of Multiple Methods in Research, 5(2), 126-149. https://doi.org/10.1177/1558689811400607

Ungar, M., \& Liebenberg, L. (2013). A measure of resilience with contextual sensitivity-The CYRM-28: Exploring the tension between homogeneity and heterogeneity in resilience theory and research. In S. Prince-Embury, \& D. H. Saklofske (Eds.), The Springer series on human exceptionality. Resilience in children, adolescents, and adults: Translating research into practice (pp. 245-255). Springer Science + Business Media. https://doi.org/10.1007/978-1-4614-4939-3_18

van Rensburg, A. C., Theron, L. C., \& Ungar, M. (2019). Using the CYRM-28 with South African young people: A factor structure analysis. Research on Social Work Practice, 291), 93-102. https://doi.org/10.1177/1049731517710326

Víllora, B., Larrañaga, E., Yubero, S., Alfaro, A., \& Navarro, R. (2020). Relations among poly-bullying victimization, subjective well-being and resilience in a sample of late adolescents. International Journal of Environmental Research and Public Health, 17(2), Article 590. https://doi.org/10.3390/ijerph17020590

West, S. G., Taylor, A. B., \&Wu, W. (2012). Model fit and model selection in structural equation modeling. In R. H. Hoyle (Ed.), Handbook of structural equation modeling (p. 209-231). The Guilford Press.

Zahradnik, M., Stewart, S. H., O'Connor, R. M., Stevens, D., Ungar, M., \& Wekerle, C. (2010). Resilience moderates the relationship between exposure to violence and posttraumatic reexperiencing in Mi'kmaq youth. International Journal of Mental Health and Addiction, 8(2), 408-420. https://doi.org/10.1007/s11469-009-9228-y

Zand, B. Z., Liebenberg, L., \& Shamloo, Z. S. (2016). Validation of the factorial structure of the Child and Youth Resilience Measure for use with Iranian youth. Child Indicators Research, 103), 797-809. https://doi.org/10.1007/s12187-016-9412-0 